\title{
Lamotrigin für Schwangere - eine sichere Option
}

\author{
Eine große Registerstudie bestätigt, das die Einnahme von Lamotrigin während der Schwangerschaft \\ das Risiko für Lippen-Kiefer-Gaumen-Spalten und Klumpfüße beim Kind nicht erhöht.
}

_ Epileptikerinnen nehmen in der Regel auch während der Schwangerschaft ihre Medikamente ein. Im Gegensatz zu Valproinsäure und Phenobarbital gilt Lamotrigin als unbedenklich in Bezug auf Missbildungen beim Ungeborenen. Eine Studie fokussierte nun auf das Risiko, dass betroffene Kinder mit Lippen-Kiefer-Gaumen-Spalten (LKG) oder Klumpfüßen geboren werden.

Die Daten stammen aus der Studie EUROCAT, in der 10,1 Millionen Geburten zwischen 1995 und 2011 registriert wurden. 226.806 Babys wiesen kongenitale Missbildungen auf. Bei der Auswertung ließen sich 147 Babys mit Lamotrigin-Exposition (Monotherapie) im ersten Trimenon finden, die nonchromosomale kongenitale Missbildungen aufwiesen. Die Odds Ratio (OR) betrug für alle LKG 1,31, isoliert für Kieferspalten betrachtet 1,69 und für Klumpfüße 1,83. Andere spezifischen Missbildungen ließen sich nicht eruieren.

Obwohl gerade das LKG-Risiko beim Kind mit der Einnahme von Antikonvulsiva signifikant stieg, lag für Lamotrigin isoliert betrachtet kein erhöhtes Risiko vor. Die Klumpfußdeformität schien nach Lamotrigin-Exposition leicht, aber nicht signifikant erhöht. Die Autoren bleiben deshalb bei ihrer positiven Einschätzung der Substanz.

- Dolk H, Wang H, Loane M et al. Lamotrigine use in pregnancy and risk of orofacial cleft and other congenital anomalies. Neurology. 2016;86:1716-25

\section{KOMMENTAR}

Die Studie bestätigt viele andere Daten, die Lamotrigin als absolut vertretbares Medikament in der Schwangerschaft iodentifizieren. Die Frage nach vermehrten $L K G$, wie sie vor allem unter Topiramat beschrieben werden, konnten sich genauso wenig bestätigen wie Klumpfußdeformitäten. Ein großer Nachteil ist die fehlende Information zur Dosierung, da wir aus anderen Registern wissen, dass Missbildungen ab einer Tagesdosis von $300 \mathrm{mg}$ durchaus relevant ansteigen können. Die Studie sollte auch nicht zu hektischem und stets riskantem Umstellen der Medikation bei Beginn einer Schwangerschaft führen. Auch andere Medikamente haben vertretbare Risikoprofile.

Dr. med. V. Homberg

\section{Schlimmes Ohr und rote Augen}

Ein 46-jähriger Mann litt seit etwa einem Monat unter einer schmerzhaften Schwellung beider Ohrmuscheln, geröteten Augen beidseits und einer Lichtempfindlichkeit. Auf Befragen gab er außerdem gleichzeitig auftretende Schmerzen im rechten Handgelenk an. Beide Ohrmuscheln waren gerötet, ödematös infiltriert und sehr berührungs- und druckschmerzhaft. Die Ohrläppchen blieben ausgespart (Abb. 1). Das rechte Trommelfell war entzündlich gerötet. Die augenärztliche Untersuchung ergab eine beidseitige gemischte Injektion. Nach Applikation von PhenylephrinAugentropfen entfärbten sich die geröteten Augen nicht, was für eine Skleritis sprach. In der Labordiagnostik ergaben sich keine wegweisenden Befunde. Der Patient wurde systemisch und lokal mit Glukokortikoiden und Zykloplegika behandelt, worunter sich der entzündliche Prozess im Lauf von einigen Wochen gut zurückbildete.

Die Polychondritis ist eine chronische entzündliche Erkrankung des Knorpelgewebes, die den Autoimmunerkrankungen zugeordnet wird. Sie kann an allen Knorpel enthaltenden Körperregionen auftreten. Besonders häufig sind Ohr- und Nasenregion betroffen, selten können auch der Kehlkopf, die Herzklappen und die Skleren beteiligt sein.

Prof. Dr. med. H. S. FüeßI

- Rodriguez-Colon G, Palestine AG (alan.palestine@ucdenver.edu).Polychondritis with auricular and ocular involvement. N Engl J Med. 2015;373:2562
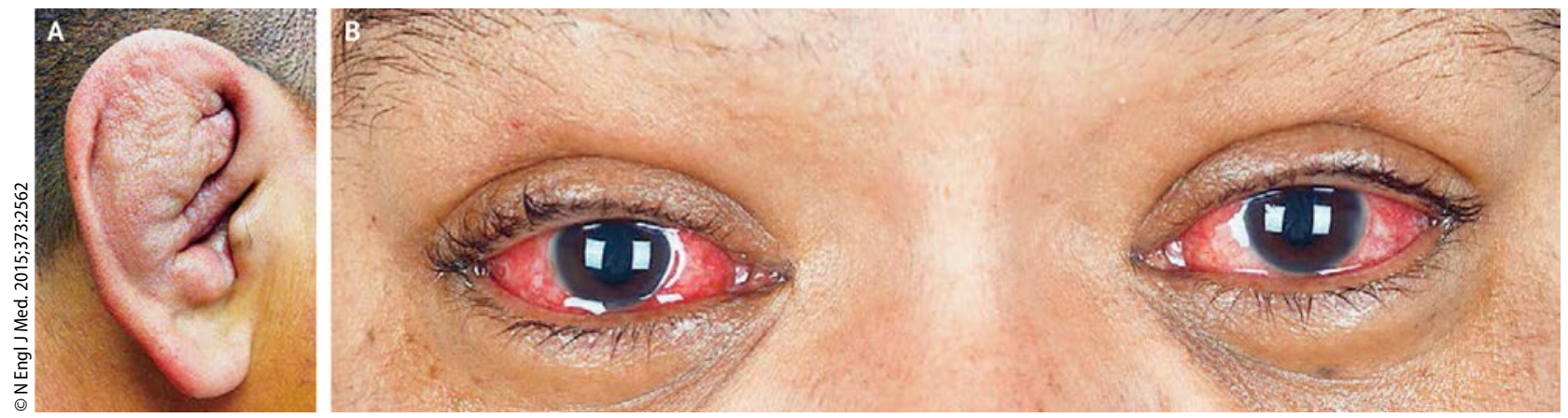

A: Schmerzhafte Schwellung der Ohrmuschel. B: Gerötete Augen. 